\title{
HIDROCONDICIONAMENTO E BIOFILMES NA CONSERVAÇÃO DE SEMENTES DE Poncirus trifoliata
}

\author{
Kelli Pirola ${ }^{1}$, Alexandre Luis Alegretti ${ }^{1}$, Marcelo Dotto ${ }^{1}$, Juliana Cristina Radaelli ${ }^{1}$, Américo \\ Wagner Júnior ${ }^{1}$
}

1 Universidade Tecnológica Federal do Paraná - UTFPR, E-mail: kelli_pirola1@hotmail.com, alexandreluisalegretti@yahoo.com.br,marcelodotto@hotmail.com,julianaradaelli@gmail.com, americowagner@utfpr.edu.br

\section{RESUMO}

O objetivo deste estudo foi avaliar a viabilidade das sementes de Poncirus trifoliata hidrocondicionadas e revestidas com biofilmes para armazenamento em duas condições de temperatura. A aplicação de biofilme à base de fécula de mandioca mostrou-se viável para sementes hidrocondicionadas de quando armazenadas até 30 dias. Para o desenvolvimento das plântulas deve-se manter as sementes em ambiente natural por até 30 dias. Recomenda-se o uso imediato das sementes para não comprometer emergência e vigor.

Palavras-chave: Poncirus trifoliata, propagação sexuada, armazenamento

\section{HYDROPRIMING AND BIOFILM IN SEED OF Poncirus trifoliata}

\begin{abstract}
This study evaluates the viability of Poncirus trifoliata seeds hidropriming and coated with biofilms for storage at two temperature conditions. The application of biofilms cassava starch base proved to be feasible for hidropriming seeds when stored up to 30 days. For the development of seedlings one should keep the seeds in natural environment for 30 days. We recommend the immediate use of the seeds for maintaing its emergence and vigor.
\end{abstract}

Keywords: Poncirus trifoliata, sexual propagation, storage

\section{INTRODUÇÃO}

A citricultura é atividade de extrema importância para o setor econômico brasileiro, produzindo metade do suco de laranja consumido mundialmente (NEVES et al., 2010). Neste sentido, quando se pensa em obter frutos de boa qualidade e manter a competitividade brasileira no mercado internacional, recomenda-se o uso de mudas certificadas, com garantias de qualidade genética e fitossanitária (OLIVEIRA \& SCIVITARRO, 2004). Todavia, ainda existem algumas dificuldades na produção 
destas mudas, principalmente relacionadas à produção de porta-enxertos.

$\mathrm{Na}$ citricultura, têm-se a opção de inúmeros porta-enxertos, cada qual com suas potencialidades, como, a tangerineira 'Sunki' (Citrus sunki Hort. ex Tan.), tangerineira 'Cleópatra' (Citrus reshni Hort. ex Tan.), citrangeiro 'Carrizo' [Citrus sinensis (L.)], citrangeiro 'Troyer' [Poncirus trifoliata (L.)], citrumeleiro 'Swingle' [Citrus paradisi Macf. X Poncirus trifoliata (L.) Raf.], tangeleiro 'Orlando' (Citrus paradisi $\mathrm{x}$ Citrus reticulata), limoeiro 'Rugoso' e limoeiro 'Volkameriano' (Citrus volkameriana) (FEPAGRO, 1995; CESM, 1998; OLIVEIRA et al., 2008). Porém, dentre estes, um dos mais utilizados no Sul do país, é o Poncirus trifoliata (L.) Raf., pois suas características de tolerância ao frio (OLIVEIRA et al., 2003), além da tolerância ao vírus da tristeza (CASTLE, 1987; HERRERO et al., 1996), o tornam atrativos.

O P. trifoliata (L.) Raf., possui sementes classificadas como recalcitrantes e apresentam tegumento mais coriáceo do que as sementes dos demais porta-enxertos, dificultando-se a embebição em água e favorecendo para rápida perda de sua viabilidade, o que compromete seu processo germinativo (OLIVEIRA et al., 2003). Assim, devem ser buscadas alternativas que permitam conservá-las por maior período, sem afetar a viabilidade de sementes, além do fato de ter-se a rápida germinação quando semeadas.

Dentre as técnicas de possível aplicabilidade, têm-se aquelas que revestem as sementes com biofilmes, contribuindo para aumentar o tempo de viabilidade das mesmas, uma vez que reduzem a atividade metabólica das sementes pela limitação das trocas gasosas com o meio externo (ALEGRETTI et al., 2015). Em sementes de jabuticabeira (Plinia trunciflora) revestidas com biofilme à base de quitosana ou fécula de mandioca os resultados foram promissores (HOSSEL et al., 2013). Contudo, para adotá-los na produção de porta-enxertos de $P$. trifoliata deve-se primeiro testá-las.

Além disso, autores como Soetisna et al. (1985), sugerem a existência de algum tipo de dormência nas sementes desta espécie, relacionando-a ao seu tegumento, atuando como barreira física à embebição de água ou à difusão de gases. Das técnicas que vem sendo aplicadas no tegumento das sementes com este comportamento, tem-se o uso dos tratamentos físicos, como do hidrocondicionamento que vem se mostrando viável para espécies como, visgueiro (Parkia pendula) (PINEDO \& 
FERRAZ, 2008) e araticum de terra fria (Annona emarginata (Schltdl.) H.Rainer) (GIMENEZ, 2012).

O hidrocondicionamento, permite hidratar as sementes previamente, preparando-se seu metabolismo para o processo de germinação sem a emissão da raiz primária (HEYDECKER et al., 1975) podendo ser utilizado para sua conservação seguida de técnica que impeça a germinação quando armazenada. Com isso o biofilme, associado após o hidrocondicionamento, pode se mostrar protocolo interessante para uso em $P$. trifoliata, principalmente por ser recalcitrante.

O objetivo deste estudo foi avaliar a viabilidade das sementes de $P$. trifoliata hidrocondicionadas e revestidas com biofilmes em duas condições de temperatura durante o armazenamento.

\section{MATERIAL E MÉTODOS}

$\mathrm{O}$ estudo foi realizado em dois ambientes, sendo parte no Laboratório de Fisiologia Vegetal e parte na Unidade de Ensino e Pesquisa Viveiro de Produção de Mudas, da Universidade Tecnológica Federal do Paraná - Campus Dois Vizinhos (PR).

Foram elaborados dois experimentos, com o primeiro submetendo as sementes ao hidrocondicionamento e o segundo sem este tratamento. As sementes utilizadas para ambos os experimentos foram oriundas da mesma planta.

Após a extração das sementes do porta-enxerto $P$. trifoliata, estas foram hidrocondicionadas em água destilada durante 48 horas. Decorrido esse tempo, as sementes foram divididas em três lotes, sendo o primeiro revestido com biofilme de fécula de mandioca $(3 \% \mathrm{~m} / \mathrm{v})$, o segundo com biofilme de gelatina e o último não sofreu qualquer tipo de revestimento. Em seguida, as sementes de cada lote foram divididas em dois sublotes, sendo um mantido em câmara fria em temperatura de $5 \pm 1^{\circ} \mathrm{C}$ e outro em condições naturais (temperatura média de $25^{\circ} \mathrm{C}$ ). As sementes de cada lote com seu respectivo sublote foram mantidas armazenadas durante três períodos (0, 30 e 60 dias).

De acordo com cada período, as sementes foram semeadas em areia na profundidade de $1,5 \mathrm{~cm}$, utilizando-se canteiros de $2 \times 2 \mathrm{~m}$. Os canteiros foram irrigados diariamente após semeadura, sempre verificando a condição de umidade do substrato. O delineamento experimental foi em blocos ao acaso, em esquema fatorial $3 \times 2 \times 3$ (biofilme $x$ ambiente de armazenamento $\mathrm{x}$ tempo de 
armazenamento), com 4 repetições de 50 sementes por unidade experimental.

Aos 110 dias após a semeadura, avaliou-se a porcentagem de emergência, comprimentos de raiz, da parte aérea e total e o número de folhas. Para emergência considerou-se o surgimento visível dos cotilédones no substrato. Os dados foram submetidos à análise de variância e ao teste de comparação de médias de Tukey $(p \leq$ 0,05). Os dados da porcentagem de emergência foram transformados previamente em arco seno $\sqrt{x / 100}$, conforme necessidade demonstrada pelo teste de normalidade de Lilliefors. As análises estatísticas foram realizadas no software SANEST $^{\circledR}$ (ZONTA \& MACHADO, 1984).
No segundo experimento aplicaramse os mesmos procedimentos adotados no primeiro experimento, com exceção do hidrocondicionamento.

\section{RESULTADOS E DISCUSSÃO}

A interação biofilme $\mathrm{x}$ ambiente de armazenamento $\mathrm{x}$ tempo de armazenamento; ambiente de armazenamento $x$ tempo de armazenamento não se apresentaram estatisticamente significativas para as variáveis analisadas. Contudo, o uso de biofilmes $\mathrm{x}$ tempo de armazenamento influenciou significativamente a emergência das plântulas e o comprimento de raiz das mesmas (Tabelas 1 e 2, respectivamente), não ocorrendo o mesmo para as demais variáveis.

Tabela 1. Emergência de plântulas de $P$. trifoliata de acordo com o biofilme aplicado na semente e o tempo de armazenamento da mesma, Dois Vizinhos - PR, 2013.

\begin{tabular}{cccc}
\hline \multirow{2}{*}{ Biofilme } & \multicolumn{3}{c}{ Tempo (Dias) } \\
\cline { 2 - 4 } & 0 & 30 & 60 \\
\hline Sem Biofilme & $94,65 \mathrm{a} \mathrm{A}$ & $29,40 \mathrm{~b} \mathrm{~B}$ & $0,12 \mathrm{a} \mathrm{C}$ \\
Gelatina & $87,18 \mathrm{a} \mathrm{A}$ & $49,76 \mathrm{a} \mathrm{B}$ & 0,36 a C \\
Fécula & $64,07 \mathrm{~b} \mathrm{~A}$ & $55,02 \mathrm{a} \mathrm{A}$ & $1,66 \mathrm{a} \mathrm{B}$ \\
\hline
\end{tabular}

CV (\%) 25,15

*Letras minúsculas na mesma coluna e maiúsculas na linha diferem entre si estatisticamente pelo Teste de Tukey (p $=0,5)$.

Tabela 2. Comprimento de raiz de $P$. trifoliata de acordo com o biofilme aplicado na semente e o tempo de armazenamento da mesma, Dois Vizinhos - PR, 2013.

\begin{tabular}{cccc}
\hline \multirow{2}{*}{ Biofilme } & \multicolumn{3}{c}{ Tempo (Dias) } \\
\cline { 2 - 4 } & 0 & 30 & 60 \\
\hline Sem Biofilme & $3,90 \mathrm{a} \mathrm{B}$ & $8,50 \mathrm{a} \mathrm{A}$ & $1,6 \mathrm{~b} \mathrm{C}$ \\
Gelatina & $7,07 \mathrm{a} \mathrm{A}$ & $6,50 \mathrm{a} \mathrm{A}$ & $2,48 \mathrm{ab} \mathrm{B}$ \\
Fécula & $5,94 \mathrm{a} \mathrm{A}$ & $7,43 \mathrm{a} \mathrm{A}$ & $6,2 \mathrm{a} \mathrm{A}$ \\
\hline CV $(\%)$ & & 59,52 &
\end{tabular}

*Letras minúsculas na mesma coluna e maiúsculas na linha diferem entre si estatisticamente pelo Teste de Tukey (p $=0,5)$. 
Quanto a emergência das plântulas de $P$. trifoliata obteve-se as maiores médias para sementes não armazenadas (tempo 0) sem revestimento e com gelatina em comparação àquelas armazenadas durante 30 e 60 dias (Tabela 1). Por outro lado, quando se fez uso do revestimento das sementes com fécula de mandioca houve possibilidade de armazená-las com mesma superioridade de emergência até 30 dias, uma vez que se assemelharam estatisticamente com aquelas não armazenadas (Tabela 1). Analisando-se a emergência das plântulas em cada tempo verificou-se superioridade para aquelas sem revestimento e com uso de gelatina no tempo 0 e para o uso de ambos os biofilmes (gelatina e fécula de mandioca) quando armazenadas até 30 dias. O mesmo não foi observado aos 60 dias já que as médias assemelharam-se estatisticamente entre si (Tabela 1).

Os biofilmes, quando aplicados em sementes, consistem na deposição de fina e uniforme camada sobre a superfície das mesmas (DUAN \& BURRIS, 1997), e estes podem regular a entrada de água e oxigênio necessários à germinação das sementes (MENEZES, 2003). Desta forma, verificouse durante os 30 dias de armazenamento essa vantagem, uma vez que, sementes revestidas tiveram médias superiores, sugerindo-se que houve maior retenção de umidade e menor atividade respiratória mantendo-se assim as sementes viáveis.

Para o comprimento de raiz verificou-se superioridade das sementes revestidas com gelatina e fécula de mandioca quando armazenadas aos 60 dias. O mesmo não ocorreu aos 0 e 30 dias, uma vez que o uso de revestimento ou não assemelharam-se estatisticamente entre si (Tabela 2). Este fato também foi observado para essa variável, nas sementes revestidas com fécula de mandioca nos três períodos de armazenamento. Por outro lado, sementes não revestidas tiveram as maiores médias para o comprimento da raiz aos 30 dias. Para gelatina, os maiores comprimentos de raiz foram nos tempos de 0 e 30 dias de armazenamento (Tabela 2). Isso comprova o fato de que o biofilme mantém as sementes com maior atividade metabólica, permitindo manter maior reserva para posterior crescimento e desenvolvimento das plantas.

Quanto ao número de folhas de $P$. trifoliata dentro do ambiente de armazenamento, as maiores médias foram com plântulas provenientes de sementes mantidas em condições naturais (Tabela 3).

Já dentro do fator tempo de armazenamento, o maior número de folhas e comprimento da parte aérea das plântulas 
foram com sementes não armazenadas ou quando mantidas até 30 dias (Tabela 4), o que comprova a hipótese da maior quantidade de reservas nestas sementes em comparação àquelas mantidas até 60 dias, pois estas últimas necessitaram aumentar seu consumo para sua sobrevivência. $\mathrm{O}$ mesmo fato ocorreu com sementes armazenadas de cerejeira-do-mato (Eugenia involucrata) (ALEGRETTI et al. 2015), sendo que neste caso as plântulas foram superiores para massa de matéria seca, aos cinco dias de armazenamento das sementes, em comparação com aquelas dos 30 dias, em decorrência do maior consumo das reservas das sementes armazenadas por 30 dias.

Os coeficientes de variação muito alto (acima de 50\%) devem-se ao fato da alta variabilidade entre as médias. Pelos resultados obtidos não houve interação significativa para biofilme $\mathrm{x}$ ambiente de armazenamento x tempo de armazenamento; biofilme $\mathrm{x}$ ambiente de armazenamento; biofilme $\mathrm{x}$ tempo de armazenamento $\mathrm{e}$ ambiente de armazenamento $\mathrm{x}$ tempo de armazenamento, bem como, para cada fator isolado nas variáveis comprimentos de raiz e da parte aérea e o número de folhas.

Tabela 3. Número de folhas de $P$. trifoliata de acordo com o ambiente de armazenamento da semente, Dois Vizinhos - PR, 2013.

\begin{tabular}{|c|c|c|}
\hline \multicolumn{2}{|c|}{ Armazenamento } & Número de folhas \\
\hline \multicolumn{2}{|c|}{ Ambiente } & $8,64 \mathrm{a}$ \\
\hline \multicolumn{2}{|c|}{ Geladeira } & $6,57 \mathrm{~b}$ \\
\hline \multicolumn{2}{|c|}{$\mathrm{CV}(\%)$} & 54,62 \\
\hline \multicolumn{3}{|c|}{ *Letras minúsculas na mesma coluna diferem entre si estatisticamente pelo Teste de Tukey $(\mathrm{p}=0,5)$. } \\
\hline \multicolumn{3}{|c|}{$\begin{array}{l}\text { Tabela 4. Número de folhas e comprimento da parte aérea de } P \text {. trifoliata de acordo com o temp } \\
\text { de armazenamento da semente, Dois Vizinhos - PR, 2013. }\end{array}$} \\
\hline Tempo (Dias) & Número de folhas & Comprimento da parte aérea $(\mathrm{cm})$ \\
\hline 0 & $9,37 \mathrm{a}$ & $7,97 \mathrm{a}$ \\
\hline 30 & 9,99 a & $6,57 \mathrm{a}$ \\
\hline 60 & $3,44 \mathrm{~b}$ & $2,0 \mathrm{~b}$ \\
\hline $\mathrm{CV}(\%)$ & 54,62 & 61,50 \\
\hline
\end{tabular}

*Letras minúsculas na mesma coluna diferem entre si estatisticamente pelo Teste de Tukey $(\mathrm{p}=0,5)$. 
Contudo, a porcentagem de emergência mostrou-se significativa à interação biofilme $x$ tempo de armazenamento, tendo as maiores médias nas sementes não armazenadas (Tempo 0 dias) em comparação àquelas que permaneceram durante 30 e 60 dias, independente do revestimento. Por outro lado, analisando-se a emergência dentro do tempo 0 e de 30 dias, as maiores médias foram obtidas para sementes sem revestimento e com biofilme à base de gelatina. O mesmo não ocorreu aos 60 dias, pois as médias assemelharam-se estatisticamente entre si (Tabela 5).

Quanto ao comprimento total houve interação significativa para biofilme $\mathrm{x}$ ambiente de armazenamento $\mathrm{x}$ tempo de armazenamento (Tabela 6).

Tabela 5. Emergência (\%) de Poncirus trifoliata de acordo com biofilme e tempo de armazenamento, Dois Vizinhos - PR, 2013.

\begin{tabular}{cccc}
\hline & \multicolumn{3}{c}{ Tempo (Dias) } \\
\cline { 2 - 4 } Biofilme & 0 & 30 & 60 \\
\hline Sem Biofilme & $78,46 \mathrm{a} \mathrm{A}$ & $44,94 \mathrm{ab} \mathrm{B}$ & $0,16 \mathrm{a} \mathrm{C}$ \\
Gelatina & $84,29 \mathrm{a} \mathrm{A}$ & $53,30 \mathrm{a} \mathrm{B}$ & $0,00 \mathrm{a} \mathrm{C}$ \\
Fécula & $60,19 \mathrm{~b} \mathrm{~A}$ & $30,19 \mathrm{~b} \mathrm{~B}$ & $2,77 \mathrm{a} \mathrm{C}$ \\
\hline CV $(\%)$ & & 27,02 & \\
\hline
\end{tabular}

*Letras minúsculas na mesma coluna e maiúsculas na linha diferem entre si estatisticamente pelo Teste de Tukey (p $=0,5)$.

Tabela 6. Comprimento total de plântulas de Poncirus trifoliata de acordo com biofilme e, tempo e local de armazenamento, Dois Vizinhos - PR, 2013.

\begin{tabular}{|c|c|c|c|c|}
\hline \multirow{2}{*}{ Armazenamento } & \multirow{2}{*}{ Biofilme } & \multicolumn{3}{|c|}{ Tempo (Dias) } \\
\hline & & 0 & 30 & 60 \\
\hline \multirow{3}{*}{ Geladeira } & Sem Biofilme & $17,54 a^{1} A^{2}(a)^{3}$ & $0,0 b^{1} B^{2}(b)^{3}$ & $0,0 b^{1} B^{2}(a)^{3}$ \\
\hline & Gelatina & 15,83 a $\mathrm{A}(\mathrm{a})$ & 18,84 a $\mathrm{A}(\mathrm{a})$ & 0,0 b B (a) \\
\hline & Fécula & 16,00 a $\mathrm{A}(\mathrm{a})$ & 18,41 a $\mathrm{A}(\mathrm{a})$ & 3,45 a B (a) \\
\hline \multirow{3}{*}{ Ambiente } & Sem Biofilme & 17,54 a $\mathrm{A}(\mathrm{a})$ & 15,46 a $\mathrm{A}(\mathrm{a})$ & $4,32 \mathrm{ab} B(\mathrm{a})$ \\
\hline & Gelatina & 15,83 a $\mathrm{A}(\mathrm{a})$ & 17,65 a $A(a)$ & 0,0 b B (a) \\
\hline & Fécula & 16,00 a $\mathrm{A}(\mathrm{a})$ & 16,64 a $A(a)$ & 9,32 a $\mathrm{A}(\mathrm{a})$ \\
\hline $\mathrm{CV}(\%)$ & \multicolumn{4}{|c|}{38,55} \\
\hline
\end{tabular}

${ }^{1}$ Médias seguidas por letras minúsculas distintas na coluna diferem entre si pelo Teste de Tukey $(\mathrm{p}=0,5)$ para o fator tempo dentro de cada biofilme.

${ }^{2}$ Médias seguidas por letras maiúsculas distintas na linha diferem entre si pelo Teste de Tukey $(\mathrm{p}=0,5)$ para o fator biofilme dentro de cada tempo.

${ }^{3}$ Médias entre parênteses seguidas por letras minúsculas distintas na coluna diferem entre si pelo Teste de Tukey ( $\mathrm{p}=$ $0,5)$, para cada biofilme e tempo dentro do ambiente de armazenamento 
Analisando-se o fator tempo 0 dentro da geladeira segundo o biofilme utilizado, verificou-se semelhança estatística entre as plântulas quanto ao seu comprimento total, sendo o mesmo observado para os períodos de 0 e 30 dias dentro do ambiente natural. Quanto ao armazenamento em geladeira, somente aos 30 dias e sem uso de biofilme demonstrou diferenças estatísticas, sendo que as plântulas obtidas de sementes mantidas em condição natural foram superiores a estas, uma vez que o armazenamento nesta condição de geladeira não obteve sementes viáveis.

Segundo Oliveira et al. (2003), sob condições de temperatura e umidade do ar não controladas, as sementes de $P$. trifoliata apresentaram perda da viabilidade após 30 dias de armazenamento, sendo que, quanto maior o tempo de armazenamento, menor a viabilidade das sementes. Foi o observado no presente experimento nesta condição de armazenamento em temperatura ambiente com o sem uso de revestimento e na gelatina. Porém, com o uso de fécula de mandioca, esta viabilidade pode ser estendida por até 60 dias.

Por outro lado, as maiores médias nos tempos de 30 e 60 dias na geladeira, foram com o uso de gelatina e fécula para o período de 30 dias e somente fécula para o período de 60 dias. Para o tempo de 60 dias em ambiente natural o mesmo ocorreu com fécula e ausência de revestimento (Tabela 6). Com isso, observou-se em ambos os casos que a fécula de mandioca quando usada no revestimento destas sementes geraram melhores respostas. Conforme já verificado por Hossel et al. (2013) em sementes de jabuticabeira (Plinia trunciflora), e por Alegretti et al. (2015) em sementes de cerejeira-do-mato (Eugenia involucrata), sendo que ambas as espécies também são consideradas recalcitrantes.

Ao analisar cada biofilme dentro de geladeira percebeu-se que sementes sem biofilmes tiveram maiores médias no tempo 0 e, para gelatina e fécula de mandioca médias superiores nos tempos 0 e 30 dias. Resultados semelhantes também foram obtidos com sementes mantidas em condições naturais sem biofilme ou com uso de gelatina. Já com o uso de fécula e mantendo as sementes em condições naturais as médias entre os tempos $(0,30$ e 60 dias $)$ assemelharam-se estatisticamente entre si.

\section{CONCLUSÕES}

A aplicação de biofilme à base de fécula de mandioca mostrou-se viável para sementes hidrocondicionadas de $P$. trifoliata quando armazenadas até 30 dias sem 
comprometer o potencial germinativo. Para o desenvolvimento das plântulas deve-se manter as sementes em ambiente natural, não ultrapassando 30 dias de armazenamento.

Para sementes de $P$. trifoliata recomenda-se uso imediato para não comprometer sua emergência e posterior vigor, independente do revestimento; porém caso se deseje armazená-las esse período não deve ultrapassar a 30 dias, devendo-se utilizar algum biofilme, independente do ambiente.

\section{REFERÊNCIAS}

ALEGRETTI, A.L.; WAGNER JÚNIOR, A.; BORTOLINI, A.; HOSSEL, C.; ZANELA, J.; CITADIN, I. 2015. Armazenamento de sementes de cerejasdo-mato (Eugenia involucrata) DC. submetidas ao recobrimento com biofilmes e embalagem a vácuo. Revista Ceres, Viçosa, v.62, p.124-127.

CASTLE, W.S. 1987. Citrus rootstocks. In: Rom RC \& Carlson RI (Ed.). Rootstock for fruit crops. New York: Wiley. p.361399.

CESM, Comissão Estadual de Sementes e Mudas do Estado do Rio Grande do Sul. 1998. Normas e padrões de produção de mudas de fruteiras para o Estado do Rio Grande do Sul. Porto Alegre: CESM. 100p.

DUAN, X.; BURRIS, J.S. 1997. Seed physiology, production e technology. Crop Science, Madison, v.37, p.515-520.

FEPAGRO, Fundação Estadual de Pesquisa Agropecuária. 1995. Recomendações técnicas para a cultura de citros no Rio
Grande do Sul. Porto Alegre, 76 p. (Boletim técnico, 3).

HERRERO, R.; ASÍNS, M.J.; CARBONELL, E.A.; NAVARRO, L. 1996. Genetic diversity in the orange subfamily Aurantioideae. I. Intraspecies and intragenus genetic variability.Theoretical and Applied Genetics, Berlin, v.92, p.599-906.

HEYDECKER, W.; HIGGINS, J.; TURNER, Y.J. 1975. Invigoration of seeds? Seed Science and Technology, Zurich, v. 3, p.881-888.

HOSSEL， C.; OLIVEIRA， J.S.M.A.; FABIANE, K.C.; WAGNER JÚNIOR, A.; CITADIN, I. 2013. Conservação e teste de tetrazólio em sementes de jabuticabeira. Revista Brasileira de Fruticultura, Jaboticabal, v.35, p.255261.

GIMENEZ, J.I. 2012. Condicionamento osmótico e GA3 na germinação de sementes de araticum de terra fria (Annona emarginata (Schltdl.) H. Rainer). Dissertação de Mestrado. Instituto de Biociências, UNESP Universidade Estadual Paulista, Botucatu. $91 \mathrm{p}$.

MENEZES, N.L. 2003. A semente e sua germinação. Santa Maria: UFSM. Acesso em: 11 ago. 2003.

NEVES, M.F.; TROMBIN, V.G.; MILAN, P.; LOPES, F.F.; CRESSONI, F.; KALAKI, R. 2010. O retrato da citricultura brasileira. Ribeirão Preto: Citrus BR, 71 p.

OLIVEIRA, R.P.; SOARES FILHO, W.S.; PASSOS, O.S.; SCIVITTARO, W.B.; ROCHA, P.S.G. 2008. Porta-enxertos para citros. Pelotas: Embrapa Clima Temperado, 45p. (Documentos, 226).

OLIVEIRA, R.P.; SCIVITTARO, W.B. 2004. Infra-estrutura e custo de produção de mudas certificadas de citros. Pelotas: Embrapa Clima Temperado, 27p. (Documentos, 118). 
OLIVEIRA, R.P.; SCIVITTARO, W.B.; RADMANN, E.B. 2003. Procedimentos para o armazenamento de sementes de Poncirus trifoliata (L.) Raf. Revista Brasileira de Fruticultura, Jaboticabal, v.25, p.461- 463.

PINEDO, G.J.V.; FERRAZ, I.D.K. 2008.

Hidrocondicionamento de Parkia pendula [Benth ex Walp]: sementes com dormência física de árvore da Amazônia. Revista Árvore, Viçosa,v.32, p.39-49.

SOETISNA, U.; KING, M.W.; ROBERTS, E.H. 1985. Germination test recommendations for estimating the viability of moist or dry seeds of lemon (Citrus limon) and lime (C. aurantifolia). Seed Science and Technology, Zurich, v.13, p.87-110.

ZONTA, E.P.; MACHADO, A.A. 1984. Sistema de Análise Estatística (SANEST). Pelotas: UFPEL, Instituto de Física e Matemática, 399p.

Recebido em: 19/10/2015 Aceito para publicação em: 26/7/2016 